\title{
Construcción de la Marca en el medio online
}

\author{
Mónica Matellanes Lazo \\ Universidad Europea Miguel de Cervantes (España) \\ mmatellanes@uemc.es
}

\section{Resumen:}

Sin lugar a dudas la Comunicación online de las marcas es de vital importancia para la visualización de sus acciones estratégicas y para conectar con los públicos más jóvenes y afines a las nuevas tecnologías. En la actualidad, dada la importancia del uso de las Redes Sociales y de lo que suponen los nuevos canales de la Web 2.0 en el ámbito de las marcas, éstas no pueden perder de vista el papel del consumidor, hoy prosumidor, en la transmisión de la imagen de marca a través de lo que dicen y comunican en la red.

Palabras clave: Web 2.0; Marcas; Comunicación online; Redes Sociales; Jóvenes.

\section{Branding online}

\begin{abstract}
:
Communication online is very important for visual strategies brands and to connect with young target who appreciate new technologies.

Nowadays, Social Networks and new channels in Web 2.0 are essential to brands so they can't forget the consumer, prosumidor called today, for communicating their online reputation.
\end{abstract}

Key Words: Web 2.0; Brands; Communication online; Networks; Young.

\section{Referencia normalizada:}

Matellanes Lazo, M. (2013) Construcción de la Marca en el medio online. Historia y Comunicación Social. Vol. $18 \mathrm{~N}^{\circ}$ Especial Noviembre. Págs. 559-571.

Sumario: Introducción. 2. Objetivos y metodología de investigación. 3. Medición de audiencia en medios digitales. 4. Marca y comunicación online. 5. Resultados y conclusiones finales. 6. Referencias bibliográficas.

\section{Introducción}

Hoy día la Comunicación online de las marcas es de vital de importancia para la visualización de sus acciones estratégicas y para conectar con los públicos más jóvenes y afines a las nuevas tecnologías.

En la actualidad, dada la importancia del uso de las Redes Sociales y de lo que suponen los nuevos canales de la Web 2.0 en el ámbito de las marcas, éstas no pueden 
perder de vista el papel del consumidor, hoy llamado prosumidor, en la transmisión de la imagen de marca a través de lo que dicen y comunican en la red.

Los cambios en los comportamientos de comunicación digital del usuario están creando un nuevo paradigma comunicativo virtual en el que las cuestiones del branding, acciones dirigidas hacia la construcción de marca, están viéndose adaptadas al nuevo entorno que marca internet.

Es por ello que esta investigación tiene por objetivos conocer los resultados sobre la percepción de la Imagen de Marca, Interactividad mantenida con los internautas, Comunicación online y posicionamiento Seo de determinadas marcas empresariales que han aumentado su presencia online en los últimos años.

Por este motivo, se va a tratar en profundidad los aspectos teóricos más importantes al respecto.

\section{Objetivos y metodología de investigación}

Entre los objetivos fundamentales de esta investigación cabe destacar:

Conocer la percepción de los individuos sobre la comunicación y presencia online de determinadas marcas con un volumen importante de tráfico en la red.

Saber cuál de todas las marcas sometidas al análisis tiene un mejor posicionamiento Seo.

Las marcas empresariales que se han sometido al estudio y análisis web son: Monster, Hola, Iberia, Softonic, El Mundo, Fnac, Ebankinter y Lanetro.

Se han escogido estos sitios web porque según los datos del Observatorio Nacional de las Telecomunicaciones (Informe de la Ontsi de 2012) son las páginas empresariales de España que más tráfico de visitas han generado a su sitio web en el año 2012.

La metodología ha consistido en una primera revisión teórica de los conceptos básicos de la comunicación online, comportamientos del usuario y hábitos de consumo online, aplicando la técnica del análisis de contenido a estas marcas a través del conocimiento de su comunicación online y del análisis de su posicionamiento Seo en la red, para comprobar el lugar de su visualización en los motores de búsqueda y así comprobar su grado de eficacia en la construcción de su imagen de marca a través de Internet.

Estas revisiones y consultas se realizaron en tres talleres en la Universidad Europea Miguel de Cervantes de Valladolid por estudiantes de entre 22 y 25 años que cursaban la asignatura optativa de Marketing en Internet. Cada uno de estos talleres duraba alrededor de dos horas y media, en los que durante este tiempo los estudiantes realizaban un análisis de la marca en la red, viendo y comprobando su comunicación y posicionamiento online. Finalmente, las plantillas de análisis recopilaron los datos numéricos a través de Excel y se realizó una lectura de los mismos, sometiéndose 
a una posterior comparativa de las marcas tratadas para llegar a las conclusiones y recomendaciones finales sobre los objetivos marcados.

\section{Medición de audiencia en medios digitales}

Muchas de las medidas de medición de audiencia en medios digitales están pensadas desde el punto de vista de los medios tradicionales, pero la world wide web ofrece mucho más. No hay que olvidar que se trata de un medio interactivo y es por ello que ve necesario la fórmula de medidas que tengan en cuenta sus implicaciones de tipo online.

Desde esta óptica, ya existen medidas de interactividad que indican no sólo qué se ve, sino también qué se hace en la página. Dónde clikea más el visitante, con qué frecuencia y cuál es la sucesión de todo ello.

Este medio permite también una medida que sólo el marketing directo podía realizar hasta ahora, y es la medición total de los resultados de una campaña. Este último apartado ha generado una gran polémica puesto que al ser posible medir cuántas personas de las que visitan una web site, lo han hecho a través de un banner puesto por la empresa en un site concreto, y controlar qué se ha hecho durante dicha visita, algunos anunciantes han propuesto utilizar dichas medidas de interactividad como forma de pago para los banner, es decir sólo pagar por los impactos útiles en base a los objetivos de la campaña (2007: 215).

$Y$ es que Internet es un medio de convergencia, en el mismo canal conviven contenidos, anuncios, anunciantes (sus propios sitios) y puntos de venta, pero realmente $¿$ Es esto relevante? Los medios tradicionales que compiten en un canal por la audiencia, en realidad compiten por el tiempo de atención. En el caso de Internet, significa que los anunciantes compiten de forma directa con sus soportes publicitarios por el tiempo de atención, y ambos compiten con los puntos de venta. A esto habría que añadir la fragmentación de audiencias que es un hecho intrínsecamente relacionado con la aparición de nuevos canales de distribución, así como la multiplicidad de los mismos dentro de una misma plataforma.

Ante esta nueva realidad la imagen de marca del producto/servicio proyectada no está bajo el control del anunciante. En Internet la imagen de marca creada es una combinación del marketing activo y del contenido generado por la audiencia, y esto último escapa casi por completo al control de la marca. Es por ello que ve necesario medir y controlar la audiencia de estos sitios digitales con vistas a la calidad y construcción eficaz del mensaje.

Tomando como ejemplo a Englash (2002) tres son los tipos de organizaciones que se dedican principalmente a la medición de audiencia en Internet: 
Empresas de investigación que mediante su software realizan medidas sobre visitas y emiten informes. Entre las más conocidas se encuentra Nielsen Netratings, Net Count de Price Waterhause y comScore.

En segundo lugar los propios publicadores de información, que a través de un software standard realizan sus propias medidas.

Y por último se encuentran las asociaciones que básicamente mediante a través de su labor como auditores de otros medios tradicionales, se están enfocando a internet. A nivel internacional $\mathrm{ABC}$, con su homónima en España OJD, la fundación CASE dependiente de la Asociación Americana de Agencias Publicitarias y la Asociación Nacional de Anunciantes, y también la AIMC en España.

En este aspecto, Calvo y Llano (2011a) hacen un mayor hincapié en las posibilidades que ofrecen las herramientas de analítica web para gestionar, crear, orientar el sitio web y estrategias Seo hacia una mayor eficiencia y especialización de los contenidos web hacia cada uno de los internautas, posibilitando así la funcionalidad del sitio y usabilidad del mismo. De esta manera, el sitio web podría captar una mayor audiencia.

Esta analítica web consiste en grabar y analizar los datos de navegación de los usuarios en un sitio web. En un primer momento, se utilizaban los datos grabados en los servidores. Actualmente, la mayoría de las herramientas de analítica web funcionan mediante un código javascript que se inserta en el pie de página, y que recoge los datos que interesen para enviarlos a una base de datos que posteriormente se podrá consultar (Gálvez Clavijo, 2010a: 75). Las herramientas profesionales más conocidas son:

Google Analytics, Omniture SiteCatalyst, XiTi, Nedstat ,Visual Sciences, WebTrends, ClickTracks, Clickdensity, que permiten medir los principales parámetros de: Páginas vistas, usuarios únicos, visitas y tiempo de navegación principalmente.

Otros datos que manejan algunas de las herramientas de analítica web son (Gálvez Clavijo, 2010b 76-77):

Datos de clicks (clickstream) que permiten reconstruir las rutas de navegación de los usuarios así como dibujar "mapas de calor" sobre una página, mostrando los elementos con más clicks.

Euros (por ejemplo, en un sitio de comercio electrónico, el importe de una compra; en un sitio de contenido, el valor de un click en un anuncio o de la impresión de un banner).

Orígenes del tráfico: es decir, los sitios web que nos envían visitas, así como las palabras clave que los usuarios utilizan en los buscadores para llegar a la web.

Páginas de entrada: es decir, la página por la que un usuario entra en un sitio.

Páginas de salida: es decir, la página desde la cual un usuario abandona el sitio. 
Eventos del sitio web: por ejemplo, número de usuarios que se registran para recibir un newsletter, número de usuarios que hacen una búsqueda, número de formularios enviados, etc.

Abandono de formularios: número de veces que un formulario se empieza a rellenar y se abandona, así como el campo en el que se abandona el formulario.

Ribes (2007a: 36) propone más técnicas y procedimientos que permiten aprovechar los datos recolectados por una herramienta de analítica web a fin de mejorar la usabilidad de un sitio web. Los aspectos a tener en cuenta según el autor son: el ClickMap, A/B Testing, Análisis de formularios y Rutas de navegación.

El clickmap (mapa de clics) es aparentemente parecido a los mapas de calor del 2eye tracking, pero en lugar de mostrar dónde miran los usuarios, muestra dónde hacen clic los usuarios.

Algunas versiones del clickmap sólo muestran datos de clics en las zonas clicables (links, botones), como es al caso de la funcionalidad llamada site overlay de Google Analytics.

Otras herramientas como Clickdensity, sin embargo, son capaces de mostrar datos de clic en cualquier zona de la pantalla, independientemente de si tiene un link o no.

Finalmente, herramientas como Omniture SiteCatalyst pueden mostrar los ingresos que genera cada clic en cada elemento de la página. Además, es capaz de mostrar datos en tiempo real.

La utilidad del mapa de clics es evidente: de un vistazo, se puede ver dónde clican los usuarios en una pantalla determinada, y rápidamente actuar para mejorarla (Banchelli, 2005: 29).

Otro aspecto interesante es el control y análisis de formularios (Maciá y Gosende, 2011a: 85) integrados en el sitio Web. Consiste en analizar el número de veces que los usuarios dejan de rellenar un formulario en un campo concreto. Con este sistema se pueden detectar fallos como el vocabulario que utilizamos para definir ciertos campos, las validaciones automáticas de los campos (que a veces no son válidas para todos los países, por ejemplo el formato del NIF o la longitud de un número de teléfono), los campos innecesarios o que incomodan al usuario, etc.

Por último, a través de los reportes de Rutas de navegación (Ribes, 2007b), se pueden obtener datos e información relevante sobre el procedimiento, el orden y preferencia de navegación de los usuarios.

Los reportes de clickstream son quizás el elemento más potente de herramientas de analítica web avanzada como Omniture SiteCatalyst o Visual Sciences. Permite responder a preguntas como por ejemplo: ¿Por qué páginas navegan los usuarios, y en qué orden?, ¿Qué páginas visitan los usuarios dadas una página inicial y una página final determinadas?, ¿En cada paso de un proceso de compra o de registro, cuántos usuarios pasan al siguiente paso, cuántos vuelven al paso anterior o cuántos 
abandonan? ¿Los que abandonan, a dónde van?, ¿Qué rutas llevan a una página determinada? (Maciá y Gosende, 2011b: 88).

Los reportes de rutas de navegación (Ribes, 2007c) de Omniture SiteCatalyst son muy útiles para analizar qué realizan los usuarios en el sitio web y, quizás más importante todavía, qué no son capaces de realizar, seguramente debido a que la página no es usable.

Asimismo, nos ayudan a definir la arquitectura de la información y el esquema de navegación del sitio web basándose en la experiencia y preferencias de navegación de los usuarios.

Existen muchas otras técnicas de analítica web que permiten mejorar la usabilidad y funcionalidad de un sitio web como el Análisis de bounce rate, Análisis de resultados de búsqueda, Segmentación y satisfacción de los usuarios. Éste último permite agrupar a los usuarios según las tascas que realizan en el sitio web, para después ofrecer contenidos e interfaces adaptados a cada perfil (Llano y Calvo, 2011b: 79).

\section{Marca y comunicación online}

En muchas ocasiones, al hablar de marcas, cómo se crean, cómo se mantienen, cómo se relanzan...se puede escuchar la expresión 'arquitectura de marca', y es que ciertamente existen muchos paralelismos entre la construcción de una casa y la de una marca. Desde necesitar unos buenos cimientos en su nacimiento que la sujeten en el futuro, hasta mimar el último de los detalles estéticos que enamoren al posible comprador, la creación de una marca sólida, una marca valiosa, es una tarea larga y compleja.

La marca es, muy básicamente, el modo principal de identificar un producto y diferenciarlo de los demás.

Según la AMA, se puede definir como: un nombre, un término, una señal, un símbolo, un diseño o una combinación de alguno de ellos cuyo fin es identificar productos y servicios de una empresa y diferenciarlos de los competidores. Esta definición se puede completar a través de las realizadas por la RAE:

Una de ellas definida como la señal hecha en una persona, animal o cosa, para distinguirla de otra o denotar calidad o pertenencia. Otra segunda como distintivo o señal que el fabricante pone a los productos de su industria y cuyo uso le pertenece exclusivamente.

Pero como mencionamos anteriormente, esto es simplemente la base del concepto. Una marca hoy en día, es mucho más que un simple elemento diferenciador.

La marca para el comprador es un punto de referencia, algo que revela la identidad del producto, concentra la información, garantiza la oferta y reduce la incertidumbre (Kotler y varios, 1999: 12-13). 
La gente compra las marcas porque confía en ellas y está dispuesta a pagar mayores precios por sus preferidas.

Las marcas forman parte de nuestras vidas, de nuestra cultura y además de unos beneficios determinados derivados de las características, cualidades y calidades de un determinado producto, nos venden algo más. Algo que en nuestro tiempo es en muchas ocasiones incluso más decisivo e importante para el consumidor que la propia calidad del producto: en definitiva un estilo de vida.

Aún sin pensar en ellas, influyen en nuestro comportamiento y cuando tenemos que tomar una decisión como consumidor, aparecen en nuestra mente, las reconocemos y confiamos en ellas. Nos dan seguridad. Hoy en día es prácticamente imposible comprar un producto sin marca. Hasta las llamadas marcas blancas se identifican por la señal del distribuidor. Un producto no se compra únicamente por sus características funcionales sino también por los sentimientos, emociones, actitudes y experiencias que se asocian a la marca (Santesmases, 2012a: 394).

La marca, junto con los clientes, es el activo más importante de cualquier empresa. Son dos caras de una misma moneda: el valor de nuestra empresa, entendido como capacidad de generar rentabilidad actual y futura, y únicamente perdurará si aporta un valor añadido que cubra las expectativas generadas (Alet, 1996: 211-217).

La importancia de crear y contar con una marca fuerte reside precisamente en ese valor añadido que se le aporta al cliente, y por el cual éste, está dispuesto no solo a comprar nuestros productos, sino que además, no le importa pagar un porcentaje extra de su valor. Cada marca aporta unos valores diferentes al consumidor y es éste quien decide cuál se identifica más con él y con sus necesidades.

Por tanto, el valor de marca se puede definir según el Marketing Science Institute como aquel valor añadido del nombre, que es recompensado por el mercado con márgenes de beneficios o cuotas de mercado mayores. Los consumidores y o distribuidores pueden ver el valor de la marca como un activo financiero y como un conjunto de asociaciones favorables y de comportamientos. En consecuencia, el valor de una marca quedará establecido en la medida en que puede conocerse y medirse cuál es el tipo y grado de satisfacción que provoca en el consumidor, y cómo ésta afecta a sus respuestas en los planos cognitivo, afectivo y de comportamiento (Muñiz, 2010: 99).

Este valor de la marca se considera como un conjunto de activos y pasivos vinculados a la misma, su nombre y su símbolo, que incorporan o disminuyen el valor suministrado por el producto o servicio ofrecido a los clientes. Por activos o pasivos, se entienden aquellos que están realmente vinculados al nombre o símbolo de la marca, y pueden agruparse en cinco categorías: lealtad de marca, reconocimiento del nombre, calidad percibida, asociaciones de la marca adicionales a la calidad percibida, y por último, otros activos en propiedad de la marca, como pueden ser patentes, marcas registradas, etc.

Estos activos de la marca, incorporan o sustraen valor tanto a los compradores como a la empresa. Por su parte, al comprador le producen los siguientes efectos: 
- Facilitan la interpretación y procesamiento de la información sobre productos y marcas.

- Proporcionan seguridad en el proceso de decisión de compra (por experiencias anteriores o familiaridad con la marca y sus características).

- Proporcionan satisfacción por el uso.

- Mientras tanto, a la empresa las marcas la ayudan del siguiente modo (Santesmases, 2004b: 112):

- Mejorando la eficiencia y efectividad de los programas de marketing.

- Proporcionando márgenes más altos al permitir precios superiores y reducir la dependencia en las promociones.

- Fortaleciendo la lealtad de marca al incrementar la satisfacción del cliente y proporcionar razones para comprar el producto.

- Proporcionando una plataforma para crecer por medio de extensiones de marca.

- Proporcionando un apalancamiento en el canal de distribución. Al igual que los compradores individuales, el distribuidor tiene menos incertidumbre si trata con marcas probadas.

- Suministrando una ventaja competitiva que puede suponer una barrera real a la entrada de la competencia.

Como se ha visto, determinar el valor de una marca es una tarea complicada, debido principalmente a la intangibilidad de los componentes que forman ese valor. De todos modos, para tratar de medirlo y cuantificarlo de alguna manera, se han propuesto al menos cinco métodos:

- El primero de ellos sería a través del precio. El precio primado que puede soportar el nombre de la marca.

- En segundo lugar, midiendo el impacto del nombre sobre las preferencias de los competidores.

- La tercera fórmula trataría de buscar el valor de reemplazo de la marca. Es decir, estimar el coste de establecimiento del negocio y de una marca comparable.

- La cuarta vía propone medir ese valor mediante el valor de las acciones de la empresa.

- Y en último lugar, se podría medir el valor de la marca, estimando el montante de los ingresos futuros.

En resumen, conseguir crear y mantener una marca fuerte supone una elevada inversión, y además tener vistas a largo plazo, pero por norma general suele ser una inversión rentable, ya que la lealtad de marca que se consigue en los clientes es un aspecto clave para el desarrollo de cualquier negocio. Una buena prueba de la importancia que tiene en nuestros días contar con una marca consolidada, la podemos encontrar en el mercado de las imitaciones, que hoy en día, en muchos casos mueve más dinero y un mayor volumen de productos que las propias marcas auténticas. Obviamente, no es el mismo producto en cuanto a calidad, pero lo importante de ello, lo que pretendemos resaltar, es que las marcas ejercen tanta influencia en la mente 
de la gente, que incluso aquellos que no se las pueden permitir, y aun sabiendo que el producto que compran no es auténtico, y por tanto su calidad es inferior, están dispuestos a adquirir una imitación para satisfacer ciertas necesidades más bien de reconocimiento y status social que les genera esa marca.

En cambio, los autores Holloway y Robinson (1995a), también adaptan estos criterios del Marketing tradicional en su adaptación al mercado y a las nuevas tecnologías y formatos digitales. De este modo, ahora el llamado e-Marketing Mix (adaptado a Internet) se compone también de: Cliente, Comunicación, Coste psicológico y Conveniencia (Holloway y Robinson, 1995b: 234).

El marketing en Internet, el llamado e-marketing, reduce las distancias entre los mercados, potenciando así su internacionalización. Las empresas se encuentran obligadas no sólo a mirar el mercado local o nacional para su expansión, sino también a prestar atención a mercados fuera de sus fronteras (Sánchez, 2001: 23).

Los cuatro elementos del marketing - mix están interrelacionados: las decisiones que se adopten con respecto a uno de ellos, van a afectar a las acciones a planificar por las otras tres.

Como se ha podido observar, la publicidad está al servicio del Marketing, constituyendo ésta una de las herramientas básicas de la ciencia del Marketing.

La publicidad constituye una forma principal de conocimiento del producto que tienen los consumidores y la principal cualidad que se le puede atribuir es su capacidad de alcanzar a un gran número de receptores en un espacio de tiempo reducido, aunque su principal limitación es su incapacidad para actuar con autonomía sin tener en cuenta el resto de disciplinas y ciencias. La publicidad es más persuasión que información. En este aspecto, hay que hablar de los elementos denotativos y connotativos, lo emocional y racional. Signos que siempre están presentes en el mensaje publicitario en menos o mayor carga (Hernández, 2004: 23).

La publicidad es comunicación expresiva que, trata de adecuarse al sistema de valores de sus receptores. Es una comunicación ornamental, valorativa y beneficiosa, que sitúa sus afirmaciones más allá del principio de veracidad exigido a los mensajes informativos. En definitiva, la publicidad forma parte de un entretejido empresarial, económico y social de vital importancia para toda la sociedad involucrando a los anunciantes, medios, agencias y receptores fundamentalmente (Quintana, 2005: 12).

\section{Resultados y conclusiones finales}

A continuación se presentan los resultados obtenidos por las marcas analizadas. Los datos han sido calificados buenos en general ya que ninguna de las marcas sometidas al estudio ha sacado un valor numérico inferior a 5 en cada uno de los criterios evaluados. Destacan los dominios de Fnac.com, Iberia.com y Hola.com como los 
sitios web mejor valorados en cuanto a contenidos, interactividad, posicionamiento Seo y valoración global de su comunicación online.

En las siguientes tablas I y II se muestran los resultados numéricos globales por marcas. Los datos destacados son los valores más altos que se han alcanzado.

Tabla I. Resultados de Valoración Global de todas las Marcas.

\begin{tabular}{|lccccc|}
\hline \multicolumn{1}{c}{ Criterios de valoración } & FNAC & SOFTONIC & HOLA & EL MUNDO \\
\hline Posicionamiento Seo & 7,9 & 6,7 & 7 & 7,4 \\
\hline Valoración Comunicación Online & $\mathbf{8}$ & 6,95 & $\mathbf{7 , 6}$ & $\mathbf{7 , 6}$ \\
\hline & & & \multicolumn{2}{c}{ Fuente: elaboración propia } \\
\hline
\end{tabular}

Tabla II. Resultados de Valoración Global de todas las Marcas.

\begin{tabular}{|lcccc|}
\hline \multicolumn{1}{|c}{ Criterios de valoración } & EBANKINTER & IBERIA & MONSTER & LANETRO \\
\hline Posicionamiento Seo & 6,9 & 7,8 & 6,9 & 6,3 \\
\hline Valoración Comunicación Online & 7,2 & 7,8 & 7,2 & 6,5 \\
\hline
\end{tabular}

Fuente: elaboración propia

Como se ha podido ver los resultados son elevados, aunque ninguna marca llega a sobresalir con notas muy destacadas. A continuación se muestran los gráficos más representativos de los dominios mejor valorados:

Gráfico 1. Fnac.com

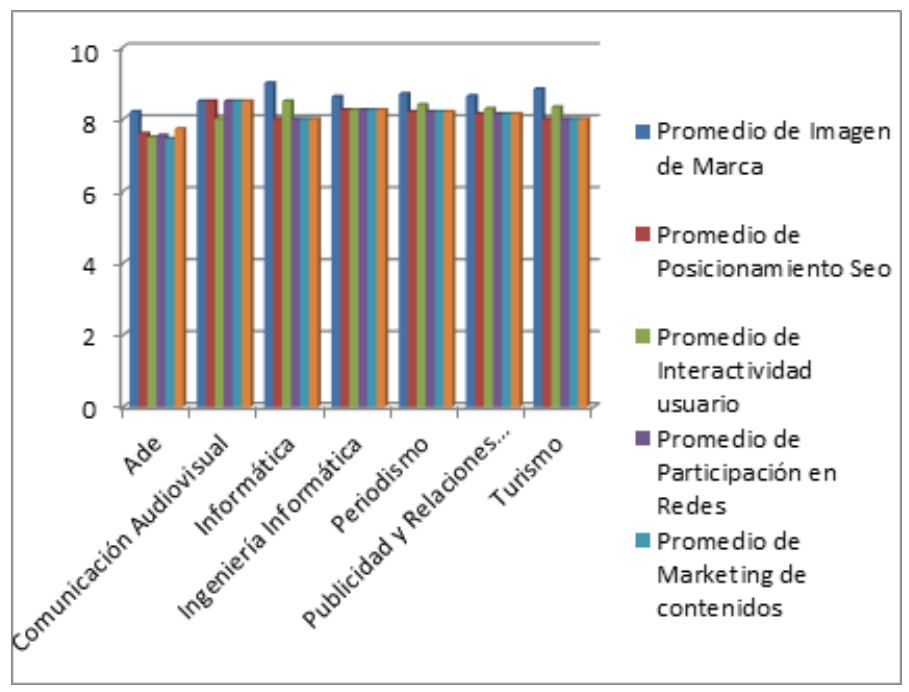

Fuente: elaboración propia 
Como puede observarse el promedio de imagen de marca es el criterio mejor valorado por los alumnos y, en este caso el valor global ha sido de un 8 , siendo la nota más alta de todas las marcas.

En el caso de Iberia.com la nota global fue de un 7,8 y de 7,6 para Hola.com. Representan resultados muy similares, en los que ninguna marca ha destacado con datos sobresalientes. La visualización de barras se ve en los siguientes gráficos, en los que puede detectarse la similitud de los datos en los criterios analizados.

Gráfico 2. Iberia.com

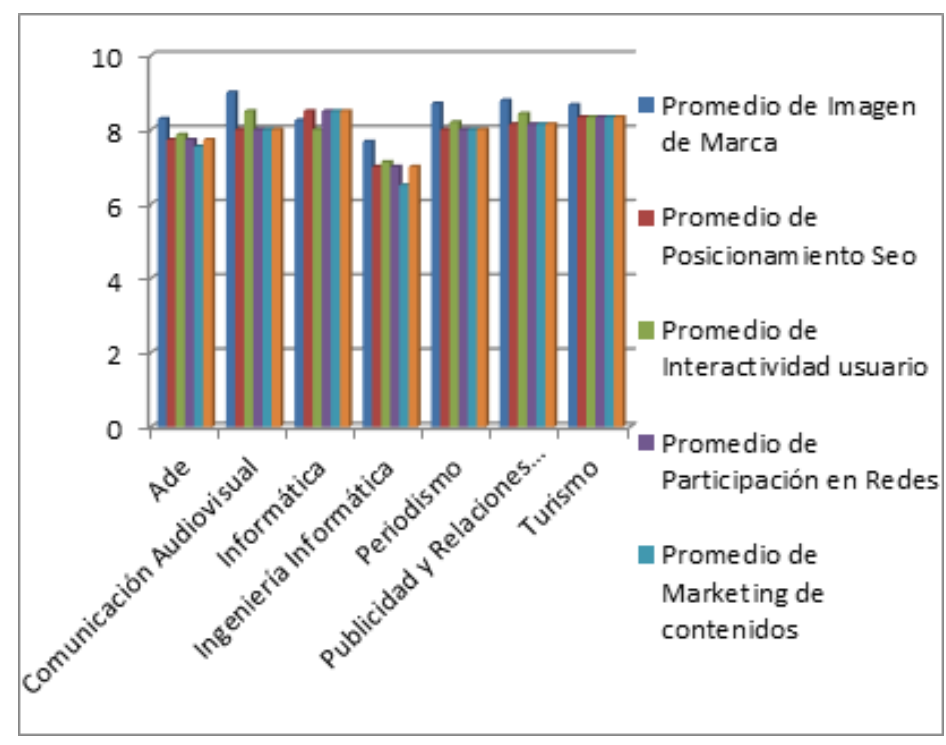

Fuente: elaboración propia

En los gráficos también puede valorarse y observarse que los estudiantes de Ciencias de la Información son los que otorgan valores muy altos en este caso, mientras que los de estudiantes de Ingeniería informática califican con valoraciones más bajas.

Otro de los aspectos a destacar es la importancia y protagonismo que cada vez se le da más al Marketing de Contenidos en la construcción de los contenidos de la página web y del blog corporativa de la marca. Es un factor destacable que debe tratarse y planificarse también con rigurosidad.

No obstante, en este sentido las marcas analizadas si cuidan este aspecto a pesar de poder mejorarlo en un futuro.

Como se ha visto, la analítica web puede y debe jugar un papel muy importante en la mejora de la usabilidad de los sitios web. Según el autor Alet (2007: 58) tres son las ventajas principales: 
Trabajar directamente con gran cantidad de datos de todos los usuarios del sitio web que permite detectar problemas de usabilidad muy específicos

Es muy fácil testear una mejora de usabilidad, así como demostrar el éxito o fracaso de un cambio de diseño (factor muy a tener en cuenta a la hora de convencer a quien toma las decisiones de que es necesario invertir en diseño o estudios de usabilidad). En un mercado en donde las aplicaciones web cada vez disponen de menos tiempo en pre-producción, la analítica web es indispensable para optimizar.

Resulta evidente considerar nuevas vías de medición de audiencias que pueden contemplar este nuevo espacio global en el que debe entrocarse modelos de negocio diferentes a los que han sustentado hasta ahora los medios tradicionales de comunicación.

Queda de manifiesto por tanto que hoy día la valoración de las marcas a través de Internet es indispensable para conseguir buena imagen de marca y alcanzar unas ventas destacables frente al resto de la competencia.

Como se ha podido destacar, es muy importante marcar una interactividad eficaz con el destinatario y realizar una potente comunicación en Social Media y varias acciones a través de toda la red; ya sea a través de su página web corporativa y de sus redes sociales.

\section{Referencias bibliográficas}

Libros

ALET, J (2007). Marketing Directo e Interactivo. Madrid: Gestión 2000.

ALET, J. (2001). Marketing Eficaz.com. Barcelona: Gestión 2000.

EGLASH, J. (2002). Cómo preparar un plan de negocios. Barcelona: Gestión 2000.

GÁLVEZ CLAVIJO, I. (2010). Introducción al Marketing en Internet: Marketing 2.0. Madrid: Ic Editorial. Madrid.

HERNÁNDEZ MARTÍNEZ, C. (2004). Manual de Creatividad publicitaria. Madrid: Ed. Síntesis.

HOLLOWAY, J. C.; ROBINSON, C. (1995). Marketing for tourism. Singapore-Australia: $3^{\text {a }}$ ed. Longman.

KOTLER, P.; CÁMARA, D.; GRANDE, I. (1999). Dirección de Marketing. Madrid: Ed.Prentice Hall.

MACIÁ DOMENE, F. (2011). Marketing con Redes Sociales. Madrid: Anaya.

MUÑIZ, R. (2010). Marketing en el s.XXI. Madrid: Centro de Estudios Financieros. QUINTANADAZA, M.A. (2005). Principios de Marketing. Bilbao: Editorial Deusto. SÁNCHEZ HERRERA, J. (2001). Plan de Marketing. Madrid: Pirámide.

SANTESMASES, M. (2004). Marketing Conceptos y Estrategias. Madrid: $5^{\mathrm{a}}$ ed. Pirámide. 
Capítulos o artículos en libros o revistas en papel

DÍAZ DE DIEGO, E. (2012). “Anunciantes españoles más creativos en BTL 2011”. En: Estrategias, n 216, Madrid: p. 10-11.

RIBES, X. (2007). "La Web 2.0, el valor de los metadatos y de la inteligencia colectiva”. En: Telos, $\mathrm{n}^{\circ}$ 73, Madrid: p. 36-43.

Artículos en publicaciones web

BANCHELLI, A. (2005). Publicidad on line. Disponible en: http://www.masternewmedia.org/es/publicidad_online/estrategias_de_publicidad/blogs_como_canales_publicitarios_efectivos_20051126.htm. [C̄onsultado el 25 de abril de 2012].

Consulta del Sitio Web del Observatorio Nacional de la Telecomunicaciones y de la SI. En: http://www.ontsi.red.es/ontsi/. [Consultado el 10 de abril de 2013].

\section{La autora}

Mónica Matellanes Lazo es Doctora en Comunicación y profesora de la Universidad Europea Miguel de Cervantes. 